\title{
Overview of the PREPARE WP3: management of contaminated goods in post-accidental situation - Synthesis of European stakeholders' panels
}

S. Charron ${ }^{1}$, S. Lafage ${ }^{2}$, E. van Asselt ${ }^{3}$, M. Baptista ${ }^{4}$, M. van Bourgondiën ${ }^{3}$, P. Brandhoff ${ }^{3}$, T. Cabianca ${ }^{5}$, J. Camps ${ }^{6}$, B. Cessac ${ }^{1}$, P. Crouail ${ }^{2}$, V. Durand ${ }^{1}$, E. Gallego ${ }^{7}$, O. Gil ${ }^{4}$, S. Holmes ${ }^{5}$, C. Hourdakis ${ }^{8}$, K. Jones ${ }^{5}$, V. Kamenopoulou ${ }^{8}$, J.F. Lecomte ${ }^{1}$, A. Liland ${ }^{9}$, I. Lopes ${ }^{4}$, M.J. Madruga ${ }^{4}$, J.O. Martins ${ }^{10}$, C. Mc Mahon ${ }^{11}$, M. Montero ${ }^{12}$, C. Murith ${ }^{13}$, G. Olyslaegers ${ }^{6}$, C. Organo ${ }^{11}$, I. Paiva ${ }^{4}$, T. Peltonen ${ }^{14}$, L. Portugal ${ }^{10}$, C. Potiriadis ${ }^{8}$, A. Prades ${ }^{12}$, M. Reis ${ }^{4}$, N. Rossignol ${ }^{6}$, T. Schneider ${ }^{2}$, R. Sala ${ }^{12}$, V. Smith ${ }^{11}$, V. Tafili ${ }^{8}$,

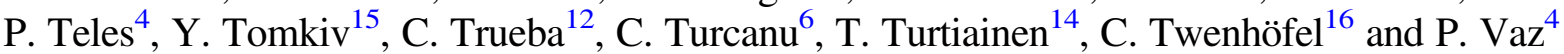

${ }^{1}$ IRSN - French Institute for Radiological Protection and Nuclear Safety, Fontenay-Aux-Roses, France.

${ }^{2}$ CEPN - Nuclear Evaluation Protection Centre, Fontenay-Aux-Roses, France.

3 RIKILT - Institute of Food Safety, Wageningen, The Netherlands.

${ }^{4}$ IST-ID - Instituto Superior Técnico, The Lisbon School of Engineering, Science and Technology, Lisbon, Portugal.

${ }^{5}$ PHE - Public Health England, Chilton, UK.

${ }^{6}$ SCK-CEN - Belgian Nuclear Research Centre, Mol, Belgium.

${ }^{7}$ UPM - Universidad Politécnica de Madrid, Madrid, Spain.

${ }^{8}$ GAEC - Greek Atomic Energy Commission, Athens, Greece.

9 NRPA - Norwegian Radiation Protection Authority, Oslo, Norway.

${ }^{10}$ APA - Agencia Portuguesa do Ambiente, Amadora, Portugal.

${ }^{11}$ EPA - Environmental Protection Agency, Dublin, Ireland.

12 CIEMAT - Research Centre for Energy, Environment and Technology, Madrid, Spain.

${ }^{13}$ FOPH - Federal Office of Public Health, Radiological Protection, Berne, Switzerland.

${ }^{14}$ STUK - Finnish Radiation and Nuclear Safety Authority, Helsinki, Finland.

15 NMBU - Norwegian University of Life Sciences, Oslo, Norway.

${ }^{16}$ RIVM - National Institute for Public Health and the Environment, Bilthoven, The Netherlands.

\begin{abstract}
Recommendations and requirements for the management of foodstuffs including drinking water and feedstuffs (but not other commodities) contaminated after a nuclear accident or a radiological event have been developed by international bodies such as Codex Alimentarius Commission or European Union as well as by individual countries. However, the experience from severe nuclear accidents (Chernobyl, Fukushima) and less serious radiological events, shows that the implementation of such systems (based on criteria expressed in activity concentration) seems to be not fully suitable to prevent several difficulties such as, for instance, stigmatization and even rejection attitudes from consumers or retailers (anticipating the fears of consumers). To further investigate the possible strategies and stakeholder expectations to deal with this sensitive issue, a study has been launched within the European research project PREPARE-WP3. The overall objective of this work, coordinated is to contribute to the development of strategies, guidance and tools for the management of the contaminated products, taking into account the views of producers, processing and retail industries and consumers. For this purpose, 10 stakeholder panels from different European countries have been set up. In addition, feedback experience from the management of contaminated goods following the Fukushima accident has been provided by Japanese stakeholders. This paper highlights the key topics tackled by the different European stakeholders' panels.
\end{abstract}

Keywords: contaminated goods management / stakeholders expectations / nuclear post-accidental situations preparedness modalities

\section{Introduction}

Recommendations and legal requirements for the management of foodstuffs including drinking water and feedstuffs as well as other goods contaminated after a nuclear accident or a radiological event have been developed by international bodies such as the FAO/WHO (Codex Alimentarius), IAEA (Safety Standards) or the European Union (EURATOM Council Regulations), and have been transposed into national Laws. 


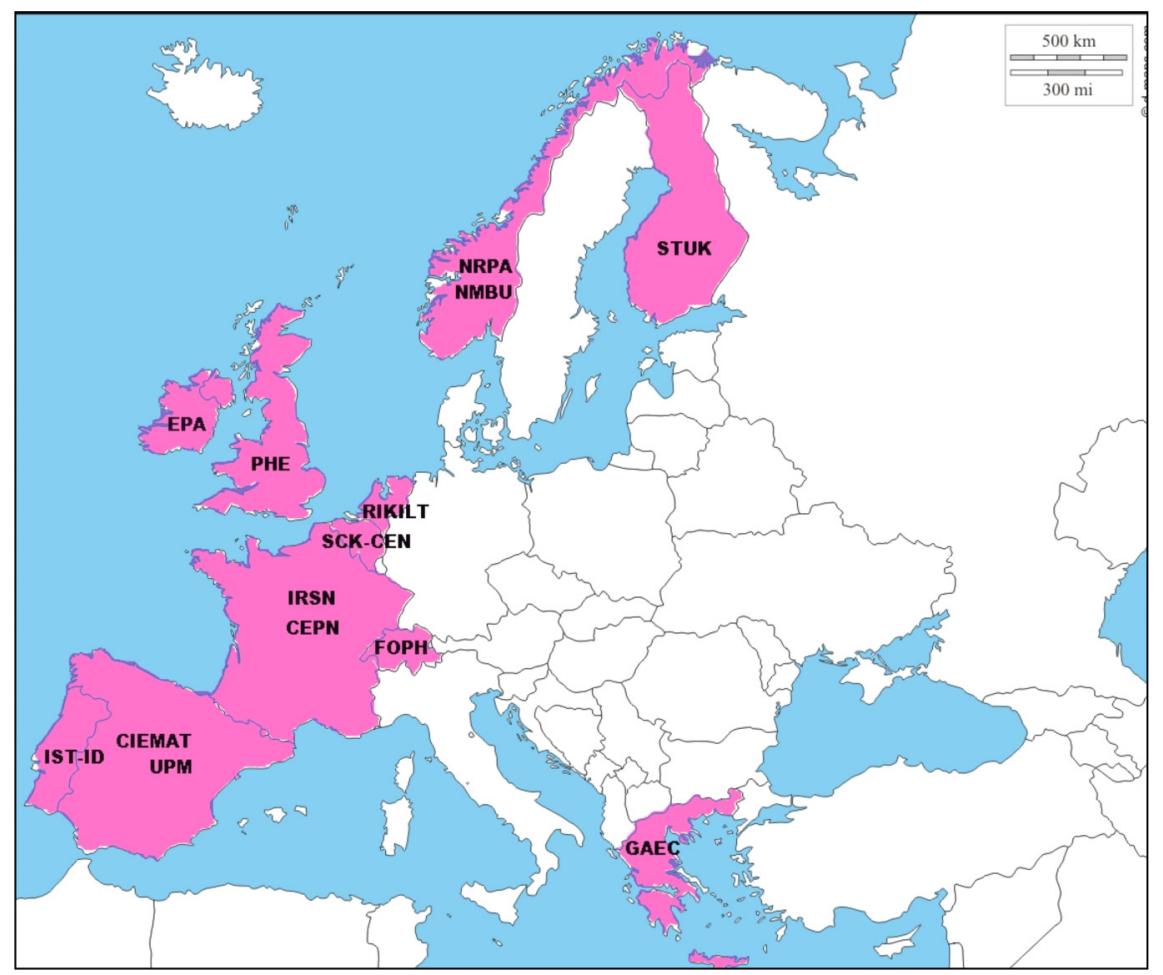

Figure 1. European countries involved in the PREPARE-WP 3 Project.

Even though such approaches provide sufficient protection for the population, the experience from severe nuclear accidents (Chernobyl, Fukushima) and less serious radiological events, shows that the implementation of such systems - most of the time based on criteria expressed in terms of activity concentration - does not seem to be fully suitable to prevent significant difficulties such as, for instance, stigmatisation of products and communities, rejection attitudes from consumers as well as from sellers and retailers anticipating the fears of their customers.

To further investigate the possible strategies and stakeholders' concerns and expectations, a reflection has been launched through a dedicated Work Package (WP3) within the European research project PREPARE. The overall objective of this work package was to contribute to the development of strategies, guidance and tools for the management of contaminated products, taking into account the views of producers, processing and retail industries and consumers. For this purpose, 10 stakeholder panels from different European countries have been set up. In addition, the feedback experience and lessons learned from the management of contaminated goods after the Fukushima accident have been provided by Japanese stakeholders.

Eleven countries through 14 organisations have been involved in the PREPARE WP3 dedicated to the management of contaminated foodstuffs and other goods, after a nuclear or radiological event (Figure 1).

Before launching the PREPARE WP3, a Working Group on Contaminated Goods ("ConGoo"), was created in May 2012 in the framework of the NERIS Platform activities. This allowed to draw a state-of-the-art of the international experiences in the management of contaminated goods after
Chernobyl and Fukushima accidents. Two thematic workshops were organised (the first one in Paris in October 2012, and the second one in Madrid, in May 2013) during which the main challenges related to the management of contaminated foodstuffs and other goods were highlighted and discussed. Most of the participants of the PREPARE WP3 attended these two workshops: it was a good opportunity to exchange information and knowledge with international organisations (e.g. IAEA, EC, FAO, NEA-OECD) and Japanese counterparts who daily face to post-accident management issues.

Three Task Force Meetings were organised in order firstly to discuss the framework and the methodology for the organisation of the stakeholders panels and secondly to exchange on the results and lessons learnt of each national panel. A final Workshop was also organised on 12-13 November 2015 in Paris to share and discuss the results in the presence of national stakeholders (consumers, producers, retailers...), international organisations (European Commission, FAO, OECD-NEA, IAEA, HERCA, ICRP) and invited Japanese colleagues (CRIEPI, University of Fukushima, producers and consumers NGO's...). The figure below shows the calendar of the different meetings and workshops (Figure 2).

This article describes the results and the conclusions of the 10 national panels presented and discussed during the Final PREPARE WP3 Workshop in November 2015 in Paris and reported in the PREPARE Dissemination Workshop organised in Bratislava on January 20-22, 2016. Furthermore, the posters presented during the Workshop in Paris and describing the methodology, the constitution of panels and the results of each national panel are presented as Supplementary Material. 


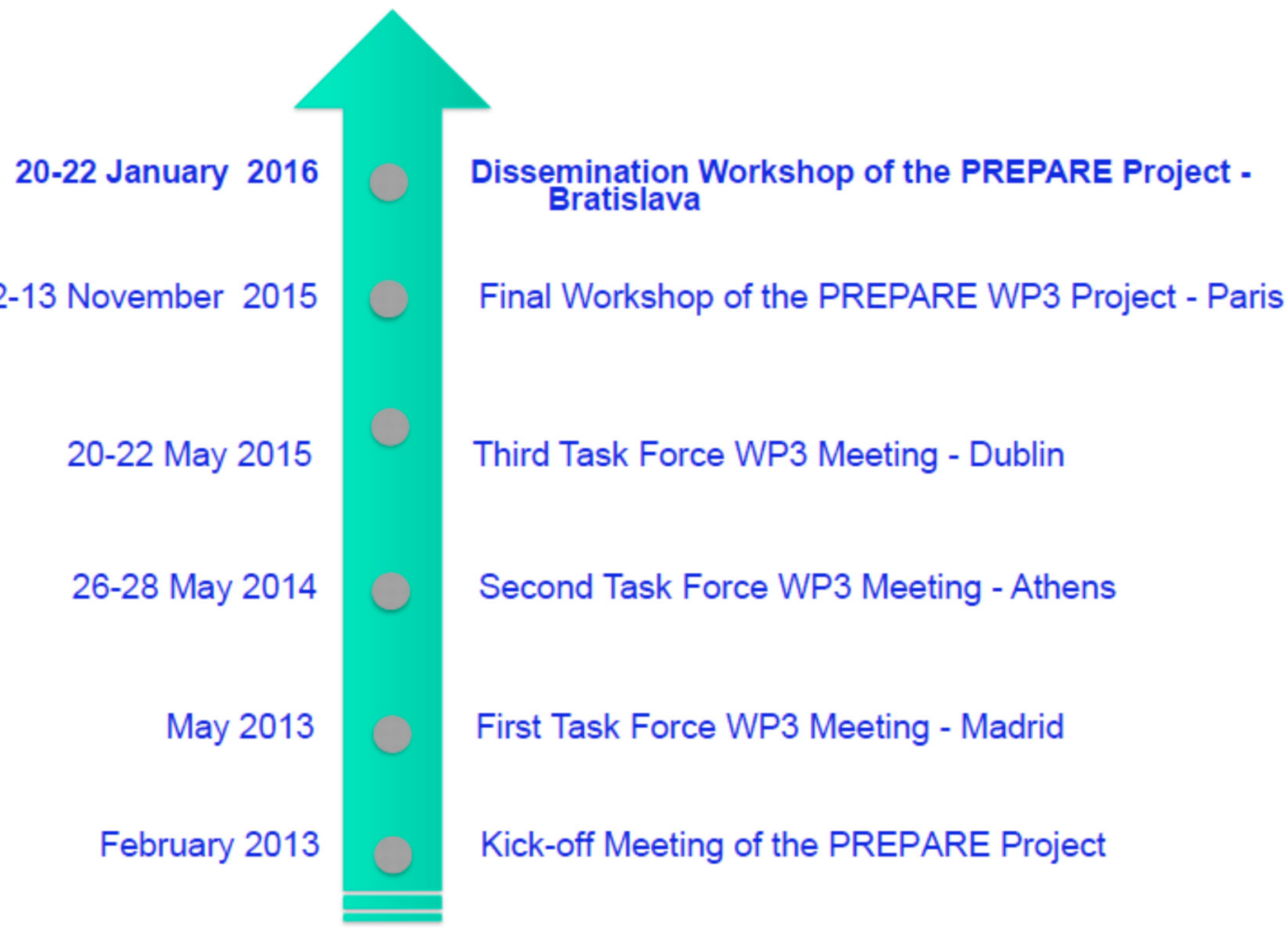

Figure 2. Calendar of the different meetings and workshops held within the PREPARE-WP 3 Project.

\section{Global organisation of the panel methodology established in each country}

Between the end of 2013 and May 2014, all participants of WP3 managed to define their own methodology for setting up a stakeholder panel in their country. The first panel meetings took place during that period, except for Norway and the Netherlands who organised their meetings in Autumn 2014.

The feedback of experiences presented by each country during the Second Task Force meeting, held in Athens in May 2014, highlighted that the establishment of national panels was successful in each country even though it is a long and intensive process. In particular, it was recognized that involvement of non-institutional stakeholders is generally difficult.

The table below summarizes the methodology adopted by each country and shows that the majority of countries focused their reflection on foodstuffs (and sometimes feedstuffs). The framework of the panel methodology was defined during the First Task Force meeting in Madrid, but each country was encouraged to adapt the flexible method to its national context. In this way, various methodological approaches were used for the composition of the stakeholder panels and the organisation of the panel meetings.

According to their specificities (e.g. nuclear vs. nonnuclear country) and past national experiences in post-accident management, different topical issues were selected and discussed during the meetings (see table below). However, it was observed that a common structure has been adopted for the organisation of each national panel meeting with:

\begin{tabular}{ll}
\hline Belgium & $\begin{array}{l}\text { Contaminated foodstuffs and other } \\
\text { consumer goods }\end{array}$ \\
Finland & Contaminated industrial products \\
\hline France \& Switzerland & $\begin{array}{l}\text { Contaminated foodstuffs } \\
\text { Contaminated ships, trucks and } \\
\text { containers, and foodstuffs }\end{array}$ \\
Greece & Contaminated foodstuffs \\
\hline Ireland & Contaminated foodstuffs/feedstuff \\
\hline Netherlands & Contaminated foodstuffs/feedstuff \\
Norway & $\begin{array}{l}\text { Contaminated foodstuffs/feedstuff and } \\
\text { other consumer goods }\end{array}$ \\
Portugal & $\begin{array}{l}\text { Contaminated foodstuffs/feedstuff and } \\
\text { other consumer goods } \\
\text { Contaminated scrap metal and other } \\
\text { consumer goods }\end{array}$ \\
\hline United Kingdom &
\end{tabular}

- a PREPARE project presentation;

- presentations of basic issues on radiological protection, regulation framework and post-accident management through table-top exercises based on NPP accident scenarios, training courses, presentations of feedback experiences of post-accidental situation from Chernobyl and Fukushima;

- discussion sessions on specific topics. 


\section{Results and national panels' lessons}

The main points tackled by the different national stakeholders have been synthesized into the five following topics:

- market, trade, economic aspects and management strategies;

- resources and capabilities, monitoring strategies;

- information strategies and decision-making process;

- management of other goods than foodstuffs and feedstuffs;

- preparedness and stakeholder participation process.

Results and outcomes from the stakeholder discussions are summarized hereafter. Prior to these findings, it must be pointed out that three key messages were particularly emphasized by stakeholders in all countries:

- everything must be done to avoid any accident. Indeed according to the panellists, citizens are victims first, and they are not responsible for the situation arising from the accident. In addition, the presence of artificial radioactivity in the environment is always illegitimate, even if the corresponding exposures are low;

- a post-accident situation would be totally new for everyone. This is so unexpected that it will lead to a loss of references and values for all the people. Upstream preparedness-before an accident occurs-is obviously crucial, but nobody will be fully ready if it happens. Only a pre-established distribution of roles of the different stakeholders will allow a quick response;

- the concept of Maximum Permitted Levels (MPLs) is useful but questionable. According to the stakeholders, MPLs are needed but their rationale is complex to understand. The definition and values of MPLs must be flexible and need to be adapted to the actual situation. They should be based on monitoring results as soon as possible, and should follow a graded improvement process.

\subsection{Market, trade, economic aspect and management strategies}

The PREPARE stakeholder panel results as well as the testimonies from Japan highlighted the complexity of a postaccident situation, which is felt as multi-dimensional: it affects the image and quality of products, it impacts local, regional, national, and international economies, generates real and potential effects on human health and has a lot of societal, cultural and ethical implications. In such a context, the policies developed for managing contaminated goods in emergency and post-accident situations have to take into account the stakeholders' concerns, expectations and values alongside scientific knowledge, as it is pointed out by recent international recommendations (e.g. ICRP109, 2009; ICRP-111, 2009; CEC, 2013).

A key issue underlined in the panels was that responsibility for the situation should not be shifted towards the citizens who are affected (as producers, consumers, etc.) by the accidental environmental contamination or threat of contamination. A post-accident situation would be new and totally unexpected and may lead to a loss of references and values for all the people. Transfer of risks, for instance through the production of wastes or the contamination of the environment should be avoided as far as possible.
Many stakeholders are concerned, from the producer to the consumer, and past experiences show the diversity of their attitudes and reactions. An appropriate management of the situation requires understanding and appropriation of countermeasures by all of them. The market is shared between short (local sale) and long (supermarkets) circuits. The panels pointed out that the consumer is always the final decisionmaker for buying goods on the market. Each consumer will react according to individual criteria. As far as food consumption is concerned, the overall quality, the taste (which is not altered by radioactivity) and the price are the most important ones. In post-accident situation, the confidence in the product (and, in the producer and the seller) is affected for a long period of time. Restoring trust (or credibility) is a long lasting and difficult process. An upstream preparedness-i.e. before an accident occurs-notably based on a previous assessment of the vulnerabilities of the potentially affected territories, is of outmost importance. In particular, it has been mentioned that the proposed policies and strategies would not be accepted by the public, if the consumer NGOs are not involved in the upstream discussion process.

According to the panellists, early and visible actions should be taken from the beginning. These actions should be rigorous, i.e. attempt to avoid residual contamination in the food chain as much as possible, but at the same time be reasonable and justified. The optimization principle of radiological protection (ALARA) should be the driving principle, taking into account not only radiological but also economic, societal, cultural and ethical aspects. The protection of the consumer is based on some key tools, such as the zoning-definition and classification of geographical areas according to the levels of contamination-and the application of the concept of Maximum Permissible Levels (MPLs) in foodstuff and feedstuff.

It has also been pointed out that the zoning criteria should be based on real measurements as soon as possible and also that they should consider the specificity of the affected areas taking into account geographical, social, environmental and nutritional background, while avoiding the creation of ineffective and counterproductive 'administrative' borders. However, for practical reasons, geographical or administrative criteria would probably be used alongside radiological criteria.

The concept of MPL provides a useful policy-support instrument. However, there are different understandings of the concept and, according to the panels, its rationale and meaning as well as the different set of levels may be questionable and confusing. The units used to express doses and activities are familiar neither to the general public, nor to the media (Turcanu et al., 2013). The name MPL itself sounds like a "black or white" concept, which does not reflect the reality. While it is a standard, which is mainly established to guarantee a safe international trade market (based on import-export conventions), it seems to be understood by the public as a level below which the consumer products are safe and above which they are surely unsafe (i.e. dangerous for human health). Consequently, MPLs appear to justify the presence of artificial radioactivity, which is however illegitimate and should not be there. Many sets of numbers are already established and their rationales as well as the methods of calculation are not easy-tounderstand. Harmonisation of the MPLs would probably be useful, at least at international level (for trade), and could 
ensure a more consistent, stable and clear system of management of contaminated goods. However, the need to have MPLs adapted to the actual situation and its evolution, in particular at the local level (i.e. in the most affected areas), has been repeatedly flagged by the stakeholders. The situation inside the affected areas (or countries) and outside is not the same. A system associating a standing set of criteria based on risk tolerability with a changing set based on quality criteria has been suggested, provided that it is justified and transparent for the consumer.

The complexity of a post-accident situation and the need to deal with previously unknown concepts can make people in the affected areas feel powerless and unable to find references for assessing the severity of the situation. The measurement of radioactivity as a way of "making it visible", and empowering people by facilitating (self-)measurements are essential elements of post-accident recovery efforts. The presentation of the Japanese experience highlighted the importance of measurements and notably the measurements made by inhabitants themselves in order to build their own reference scale and recover a grip on their daily life. This response strategy seems relevant for the panellists.

There may be some reluctance from consumers towards a residual radioactivity in food products (Turcanu and Perko, 2014). As a consequence, producers ask for a graded approach for the management of contaminated goods based on an improvement process. Such a process, including for example clean animal feeding and agricultural soil treatments (decontamination), may be efficient if it is done on a step-by-step basis. If countermeasures are proactively and transparently undertaken, these will help to regain credibility and trust. The concept of the "dilution of radioactivity" has been also discussed. This is a very sensitive issue. Even if it is totally forbidden "in a period of peace", it might be tolerable "in a period of crisis" (for instance, it has been pointed out that the reprocessing allowed to mitigate the radioactivity content in foodstuff/feedstuff in agricultural regions affected by the Chernobyl accident). However, this implies ethical considerations and could not be implemented without a process of stakeholder-wide consensus building. However, other possibilities should also be explored, such as the diversification of the diet.

A balance between the interests of the producer and consumer should be sought. If the situation is severe, compromises will have to be found between the food quality, the cost, the sustainable development of the affected areas without affecting human health, the image of the product and its production area, as well as the market competition. The waste management strategy may also be a key parameter. The presence of artificial radioactivity may be offset by the improvement of the global quality of the product. How far can one go for supporting a production or a sector? This is a crucial question. A kind of solidarity should be built, based on health, economic and ethical considerations. Solidarity from the consumers cannot exist without accountability from the producers, based on an improvement process, a rigorous monitoring strategy and transparency. It may be built using pre-existing or new stakeholder networks. Cross-border solidarities are probably more difficult to set up.

Indemnification and compensation of affected stakeholders is a key issue since it is clear that economic consequences will affect whole sectors especially for products with important export shares. It was recognized that any compensation scheme could lead to undesirable effects. In particular, it can provide inequities between individuals, create unfair market situations, and influence or even block decisions. In a long-term postaccident situation, the management of potentially contaminated food should rely on regional and inter-professional organisations, which generally have pre-established response teams and mutual aid funds.

The latter point raises the question of the difficulty to lift countermeasures, and how to justify that they are no longer necessary. As a consequence, the strategies which consist in implementing early countermeasures on a very large perimeter before reducing it according to the improvement of the situation should be prudently considered. This issue should be anticipated and discussed with stakeholders. Especially, protocols for clearing foodstuffs should be established during the preparedness phase.

\subsection{Resources and capabilities, monitoring strategies}

Regarding resources and capabilities, emergency situations can put an important stress on the economy and even cause a societal disruption. Even though governmental bodies have the knowledge of the operational procedures and the ability to deal with the follow-up operations, there are doubts regarding the national capabilities to respond to such events. There are usually enough resources to handle routine operations, but it can be overwhelming in emergency and recovery situations, since personnel and monitoring equipment are quite often limited: for instance, handling a large number of samples will be very challenging in the short term and difficult to sustain in the long term. According to the stakeholder viewpoints, there is often an unclear visibility of the competent institutions role and a lack of knowledge concerning support networks. It means that there is a need for a clarification of the roles and responsibilities of governmental bodies and for an identification of a potential non-governmental contribution.

Monitoring is a key issue in a post-accident situation. The purpose of measurements should be clear (compliance with regulations or risk estimation) and the establishment of radiation monitoring networks is fundamental to compare measurements and to properly guide protective actions. Monitoring strategies should consider: the standardisation and harmonisation of procedures for the measurement of radioactivity, the prioritization of samples, logistic and samples storage capacity, adequate training of people involved, expert guidance, accreditation and use of dedicated laboratories in industrial partners (control of contaminated goods). In the future, it could be interesting to open a dialogue with the stakeholders on the calculation assumptions and the consistency of the radiological criteria for monitoring.

Stakeholders are very sensitive to issues related to radioactive contamination monitoring and its perception depends on the way the subject is communicated. Many regulations and standards are available, but guidance on their implementation is required. It was stressed out that there is a general need and a demand for more education and training across all stakeholders, including the media. In addition, it was stressed that plurality of radiation monitoring networks is important to compare measurements and to improve the protective actions. 


\subsection{Information strategies and decision-making process}

From national stakeholder panels discussions it is clear that one of the most important issues in the event of a nuclear emergency is good communications with all stakeholders. Communication paths must be clear to avoid confusion and to ensure the public and professionals are not receiving contradictory messages. The provision of accurate, clear and transparent information is vital for building trust amongst the public and other stakeholders. The speed with which information is provided is crucial and the use of social media would play an important role that must be anticipated.

In the preparedness phase, responsibilities regarding communication should be clarified and decisions should be made about who would deliver the communication. In a nuclear crisis context, the public are more likely to trust independent health and scientific experts rather than government officials or those with vested interests in the electronuclear industry. To ensure that information is quickly provided, generic and easy-to-understand key messages, which could be tailored in the case of emergency, should be prepared in advance. As far as possible all relevant stakeholders must be involved in the communication plans as well as in emergency exercises. This allows them to build interrelationships and helps to improve communication between them. In that perspective, it is very beneficial to involve journalists in the preparedness phase as the media can influence consumer views and behaviours.

In order to design an effective information strategy, it is important to anticipate, as far as possible, the expectations and concerns of the public regarding radioactive contamination of food and other products. When an accident occurs, many official channels of information may lose their credibility (government, experts, nuclear industry...). Nevertheless, they would be expected to provide information. Intermediate channels may be activated such as medical staff personnel, teachers, NGOs and elected representatives, local agencies of agriculture, trade unions...). The plurality of the information sources and expertise is welcome. Good cooperation is needed between government and industry both in the provision of information and also in the implementation of protective actions. Food and agricultural protective actions should be developed in cooperation with those responsible for implementing them. Emergency plans should avoid as far as possible the spreading of false rumours and prevent over-reaction such as unduly rejection goods, for example only because of their origin. It should also take into account the predictable nuances in the risk perception among people, e.g. the increased concern of young or future parents. In another hand, it is important for the experts to be at the service of the population and progressively restore their credibility. It means that they have to commit themselves working at the local level together with - and not just for-the population.

In order to pass information and improve the understanding of the situation, experience shows that searching for appropriate solutions is preferable than lecturing affected people. When communicating risk, the language used should be straightforward, non-technical and the risks should be explained by comparison with daily life examples and familiar concepts. A kind of consistency should be sought for these key messages in all Member States. In addition, in case of an accident in Europe, if mixed messages are being sent outside this would have a negative impact on foreign markets. To protect exportations, the EU response would be critical.

For the professionals, guides and handbooks should also be prepared in advance. For instance, the European Handbook for Food Production Systems could be used to prepare a national catalogue of appropriate protective actions. The advantages and disadvantages of the various protective countermeasures in terms of cost, feasibility, acceptance and sustainability should be included.

According to the European Directive 2013/59/EURATOM (CEC, 2013) that has to be implemented before February 2018, each Member State should indicate which aspects are included in the decision-making process and how corrective actions are optimised. Regarding the introduction and cessation of agricultural protective actions, the communications plan must be very clear about who would communicate instructions to producers and where they can seek further information and support. It would be useful to provide them with examples of the potential effectiveness of these protective actions if they have been implemented in the past.

In the decision making process there are many aspects that need to be evaluated in a transparent manner such as health effects (which are not limited to doses), remediation costs, production of waste, technical feasibility, societal and environmental aspects, short- and long-term acceptability of the protective actions by both producers and consumers and also reassurance. The decision making process should be transparent with regard to the choices of parameters as well as the weights assigned to each one. The communication of any decisions made is extremely important for a good understanding of the situation by the public.

\subsection{Management of other goods than foodstuffs and feedstuffs}

In case of a radiological event involving radioactive releases into the environment, the contamination of feed and foodstuff can be an important contributor to doses received by the public. Therefore, the issue is addressed in emergency plans in many countries, notably on the basis of Maximum Permissible Levels (MPLs) laid down at international level (Codex Alimentarius) and European level (EU regulations). The results of the panels focussed on foodstuff showed however that the situation for such products is complex and remains difficult to deal with in case of a radiological event.

On the other hand, vast amounts of non-food products and raw materials are produced in Europe and around the globe, for which there is no clear regulation in case of a radiological event. Production is often a complex system consisting of production/recycling of raw materials, manufacturing of components, assembly of products and sales. Many stakeholders are involved such as producers, subcontractors, overseas factories, subsidiary companies, retailers and carriers. These products are continuously transported through transport hubs via road, rail, sea, inland waterways and air, within and across borders. They finally fall into the hands of end-user consumers.

Assessments and past experience (e.g. after the Fukushima accident) show that the contamination of non-food goods is 
generally not a significant exposure pathway for the population. However, such contamination should be controlled in order to protect the population and in particular workers who may be in contact with contaminated goods during production, handling and transport. If a contamination-even low-is detected at any control point, the production and delivery of the product may seriously be affected or even halted for a long time. Furthermore, goods that are contaminated above regulatory criteria, could be abandoned by their manufacturer, owner or carrier if there is no existing safe disposal to manage them as radioactive waste.

The discussion of the panels about goods other than foodstuff aimed to highlight the current legal and procedural framework relating to the management of such goods and further explore the practical issues in this area in order to establish consensus on what strategies should be developed for their management and identify what future work needs to be done.

Management of complex or extensive contamination cases can only be successful when all actors know their respective roles and responsibilities. According to the panellists, while the organisations or agencies in charge of the monitoring of goods (such as customs...) are rather well identified, those having the responsibility to manage them if they are considered as contaminated, or not. Among the other stakeholders, the manufacturer is normally responsible for the overall safety of the product. Production chains, however, are long and complex and in many cases the origin of any single component of a finished product is not known and the responsibility thus falls to the owner. The shipper may also be designated as responsible. Currently, international trade rules do not include the issue of radiological contamination in the agreements on responsibility. As a consequence, it may be difficult to determine what are the responsibilities of each stakeholder, what are the rules to be applied and who is in charge of their enforcement.

More generally, there is no specific international legislation that applies to goods contaminated after a nuclear accident. In that context, as shown by past-experience after nuclear accidents (Chernobyl and Fukushima) and other crises, the application of regulations issued for non-accident situations is attempted. However, it is not clear whether these regulatory regimes may apply in case of emergency and experience shows that they do not fit well. The panellists stressed that the priority should be to protect personnel staff against the threat for their safety - although the process for that is not clear - rather than to control the radiation levels of goods. The discussions of the panellists focused on the management of contaminated goods, which cannot be released without authorization. The owner may refuse to take back the goods and the shipper may refuse to transport them once they have been declared as radioactive; the responsibility for repackaging or disposal of these goods may then fall to the carrier. If there is no provision for the management of rejected goods, and notably no facilities for a safe disposal of them, contaminated goods could be abandoned in an inappropriate location, and thereby becoming "orphan source".

From the regulator's point of view the main challenge is, however, to determine practical criteria for the release of goods. But again, in the absence of specific criteria for contaminated goods laid down for post-accident situations, the use of some current reference values established for other purposes may be an option. These could be, for instance, the exemption and clearance levels that have been set internationally (IAEA, 2004). However, they have been established for planned exposure situations and neither for emergency nor for existing exposure situations. Further, they are based on a reference dose criteria of $10 \mu \mathrm{Svy}^{-1}$, which is much lower than those used for food $\left(1 \mathrm{mSv} \mathrm{y}^{-1}\right)$. Moreover, it is unclear whether the same criterion applies, no matter how, by whom, and for what the goods are used. There will be so many exposure scenarios to take into account that such an approach would probably be misleading. Another option is to use the surface contamination limits and standards established for the transport regulation. However, the corresponding levels are not really appropriate for a post-accident situation. The issue of contaminated goods is so diverse that it would be difficult to determine a set of numbers adapted to all cases.

Because the management of the situation depends to a large extent on the context (severity of the accident, type and number of contaminated goods, location of the control points, monitoring capabilities, etc.) the panellists considered that there was no need for an additional international legislation addressing specifically the issue of "non-food contaminated goods". In particular, they did not advocate the existence of specific criteria for release. However, they highlighted that the elaboration of guidance on how existing regulations could apply in a practical way would be useful. The panellists also agreed that, because goods are transported, both in the EU and worldwide, a global and international approach is needed to develop that guidance. In particular, a common approach to deal with orphan goods would be welcomed, especially as some countries do not have the expertise and infrastructures enabling them to manage the repatriation and storage of contaminated goods.

Monitoring strategy would be a real challenge for industries and manufacturers. During the panel discussions, it was clear that a very few number of companies and industries have included radiological protection in their crisis management plans, with the exception of, however, the steel industry, which is prepared for orphan sources with portal monitoring and management protocols. Capacity for monitoring has raised vivid discussions. Some companies would favour the setting up of a jointly operated laboratory with a high enough capacity for screening. Prospective buyers accept in-house control certificates only if the laboratory has accreditation. The companies must follow instructions given by the authorities. However, protocols and accreditation systems may vary between different countries. This may create confusion in companies, which operate in several countries. This issue should be prepared in advance and included in emergency plans, in particular for industries with overseas factories.

Like for food, the public perception is a crucial point. It is always difficult to demonstrate that very low contamination of goods still fits with heath safety principles. The panellists recommended the development of information and communication tools, preferably in advance. Predetermined points of contact could be identified or created to allow exchange between relevant authorities and potentially affected industries. Leaflets could be prepared in advance and general information should be made available to all stakeholders (agencies, industry and general public). Forwarding companies 
have huge client registers, which could be used for the circulation of the information in case of crisis.

Considering the complexity of a post-accident situation, the blur in terms of responsibilities as far as contaminated goods are concerned, the lack of protocols, criteria and guidance in that field, the panellists are aware that much remains to be done, even though it would be difficult to set universal criteria in advance which would be adapted to all situations. The panellists thus recommended continuing to explore the issue with relevant stakeholders in order to help the development of an approach shared in the European context or even beyond.

\subsection{Preparedness and stakeholder participation process}

All panellists pointed out the importance of preparedness and the need to involve all the relevant stakeholders, even if it is difficult to implement in practice. The issue is addressed in many international recommendations and standards, such as ICRP Publication 111. Some key provisions have been included in the European Directive (CEC, 2013) such as:

- the development of response plans that cover not only the emergency and transition phases but also the recovery and long-term remediation phase;

- the description of the decision-making process;

- the identification and involvement of different stakeholders in the different stages of the management system;

- the implementation of education and training programmes;

- the development of communication strategies.

The preparedness is also addressed by OECD/NEA, which recommends that the generic emergency management system (NEA, 2010) should include several stages, such as-the identification of the possible protective actions, - the allocation of resources for their implementation,-the required legal framework at the local/regional and national levels, taking into account feedback from training, exercises, audits, action plans with a reinforced stakeholders participation at each stage of the process.

During the emergency preparedness for contaminated goods, institutional and non-institutional actors should be involved in dialogue. As all the life dimensions would be affected after an accident, there would be a wide range of issues at stake: the determination of the roles and responsibilities and the coordination of potential stakeholders as well as the identification of local/regional/national vulnerabilities in case of an accident should be key elements for designing an effective emergency management system.

It is obvious that among institutional actors (e.g. direct managers and decision-makers) there are organisations, institutions and experts, which have no radiological protection background although they have specific roles and responsibilities in emergency preparedness and response. This is also the case for non-institutional actors, stakeholders affected by a nuclear/radiological accident and those affected by the decisions taken, as well as organizations involved in public information. The stakeholder participation process is necessary but complex. It is a real challenge to identify, attract, engage, keep active and coordinate all these stakeholders, mainly because:
- apart from nuclear emergency response agencies and regulatory authorities, other stakeholders lack previous experience and a radiation protection background;

- nuclear emergencies are very complex situations with important societal, economic, and political implications, involving multiple stakeholders;

- different approaches are required at each phase after a radiological event;

- various stakeholders have different needs and interests;

- all stakeholders have different roles and responsibilities but they are not familiar with others' roles and responsibilities.

The panellists raised many specific issues. In order to ensure a good coordination between different stakeholders, it is important to identify roles and responsibilities, the interaction among groups and awareness of each other's action plans. The pre-existence of local and regional inter-disciplinary networks and the balance between consumers versus producers' interests should be addressed. Guidelines and handbooks with procedures about what to do, including strategy criteria and stakeholder networking, should be developed in advance.

In general, there is a need for basic training on radiological protection issues in order to speak the same language, as well as to promote the radiological protection culture. Education and training initiatives for the technical staff in charge of the implementation of the contaminated goods management plans should routinely be established. Special training programs can also be delivered to the stakeholders, who do not have radiological protection skills.

One of the problems for the institutional stakeholders is the limited resources, notably in terms of technical capabilities. In the case of non-institutional actors, there is a lack of trained personnel to deal with radiological emergencies. Globally, the panellists expressed their doubt about the capability of countries to manage large-scale contamination of goods.

In case of a radiation emergency, European initiatives are expected in cooperation with competent international organisations (e.g. the UN Food and Agricultural Organisation, IAEA, International Maritime Organisation). The provision of assistance to countries lacking the required expertise or infrastructure for the management of contaminated goods is also expected.

Experts and media should be trained to communicate radiation-related concepts by using simple language. Communication plans should be prepared in advance, in order to (re-)build public trust. Risk communication and transparency are crucial factors: flow, content, timescales, and credibility of information are basic steps to achieve public confidence.

Finally, the experience gained from the establishment of the different PREPARE National Panels (Deliverable report 3.2 , 2015) for preparedness and stakeholder participation processes, shows that there is an added value of stakeholders participation in the management of contaminated goods. This added value can be described as follows:

- it provides an opportunity for the stakeholders to gain new knowledge on the topic of radioactive contamination and the national systems of radiological and nuclear emergency preparedness and response;

- it gives the experts and authorities an insight into the feasibility and acceptability of suggested actions in various sectors;

- it increases the networking opportunities; 
- it allows building trust and understanding between actors, which can be crucial during real events.

\section{Recommendations and perspectives}

The first result is the fruitful participation of various stakeholders in the national panels in Europe and their willingness to continue to be involved into the reflection.

The work performed with the national panels also highlighted some lessons. There is a need to favour their involvement in the reflection but also to ensure the respect of their values. The objective of involving the stakeholders is not to promote the acceptability of the accident: citizens are victims. The objective is to build trust and understanding between stakeholders. This can be crucial for managing emergency and recovery situations. In addition, to engage dialogue with stakeholders on this issue, it is important to rely as much as possible on existing structures. All stakeholders involved in the national panels also indicated that preparedness on the management of contaminated goods is crucial. Nevertheless, it is unrealistic to think that everything could be prepared in advance. The situation will be totally new and will lead to a loss of references. So, it is essential to be ready to react promptly if an accident occurs. It is also essential to develop tools that allow better predictions for emergency situations follow-up behaviours and be able to implement corrective actions to overcome loss of references and mistrust. Long-term perspectives have to be considered while implementing the actions. In addition, the significant contribution in the reflection of the feedback provided by Japanese experts and stakeholders on the follow-up of the Fukushima accident was mentioned.

The results of the national panels allowed to identify some issues needing to be further investigated:

- the responsibilities of the different actors are essential for improving the preparedness but the role of each other should be made clearer;

- concerning other goods, processes should be streamlined and approaches for other goods should be consistent with food;

- compensation schemes have to be considered and put into debate as they play a key role (positively and/or negatively) in the management of the contaminated goods;

- in the process of restarting the distribution of goods after an accident, the role of local networks has to be investigated;

- challenging issues on traceability, brand image and the distribution of the goods on national and international markets;

- the role of social media and the importance of communication strategies;

- the promotion of education and training on this issue and the development of the radiological protection culture among the different stakeholders;

- the opportunity to open a dialogue with the stakeholders on the calculation assumptions and the consistency of the radiological criteria for managing the situation in order to deal with conflicting criteria and favour their understanding and usefulness on one hand and on the other hand to explain the possible evolution of the response strategies and the long term perspective at the beginning, based on the accident in order to derive robust management options.

Some proposals were discussed to continue the reflection. The first one is to promote the diffusion of the results through the report including feedback to national stakeholder panels. In addition, the results could be presented in order to share lessons learned with national and international organisations. The second one is to continue the dialogue by keeping the contact with the national panels. For that, it is proposed to ensure a coordination within the NERIS "ConGoo" (Contaminated Goods) working group. Likewise, the cooperation with Japanese experts could be reinforced to draw lessons from the follow-up of the management of the post-accident situation in Fukushima.

\section{Supplementary Material}

Posters presented during the Workshop in Paris. The Supplementary Material is available at http://www.radiopro tection.org/10.1051/radiopro/2016038/olm.

Acknowledgments. The authors wish to thank all the stakeholders involved in the 10 national panels for their active contribution.

\section{References}

CEC (2013) Council Directive 2013/59/Euratom of 5 December 2013 laying down basic safety standards for protection against the dangers arising from exposure to ionising radiation, and repealing, Directives 89/618/Euratom, 90/641/Euratom, 96/29/Euratom, 97/ 43/Euratom and 2003/122/Euratom.

IAEA (2004) Application of the Concepts of Exclusion, Exemption and Clearance, Safety Guide No. RS-G- 17.

ICRP-109 (2009) Application of the Commission's recommendations for the protection of people in emergency exposure situations. ICRP Publication 109, Ann. ICRP 39 (1).

ICRP-111 (2009) Application of the Commission's recommendations to the protection of people living in long-term contaminated areas after a nuclear accident or a radiation emergency. ICRP Publication 109, Ann. ICRP 39 (3).

NEA No. 6387 (2010) Strategic Aspects of Nuclear and Radiological Emergency Management.

Turcanu C., Perko T. (2014) The SCK•CEN Barometer 2013. Perceptions and attitudes towards nuclear technologies in the Belgian population, Open Report of the Belgian Nuclear Research Centre BLG-1097, Mol: Belgium.

Turcanu C., Perko T., Geenen D., Aerts H., Goussarov G., Vermeersch L. (2013) Media reporting on food contamination after the Fukushima accident. Content analysis of four Belgian newspapers, Open Report of the Belgian Nuclear Research Centre BLG-1095, Mol: Belgium.

Cite this article as: S. Charron, S. Lafage, E. van Asselt, M. Baptista, M. van Bourgondiën, P. Brandhoff, T. Cabianca, J. Camps, B. Cessac, P. Crouail, V. Durand, E. Gallego, O. Gil, S. Holmes, C. Hourdakis, K. Jones, V. Kamenopoulou, J.F. Lecomte, A. Liland, I. Lopes, M.J. Madruga, J.O. Martins, C. Mc Mahon, M. Montero, C. Murith, G. Olyslaegers, C. Organo, I. Paiva, T. Peltonen, L. Portugal, C. Potiriadis, A. Prades, M. Reis, N. Rossignol, T. Schneider, R. Sala, V. Smith, V. Tafili, P. Teles, Y. Tomkiv, C. Trueba, C. Turcanu, T. Turtiainen, C. Twenhöfel, P. Vaz. Overview of the PREPARE WP3: management of contaminated goods in post-accidental situation-Synthesis of European stakeholders' panels. Radioprotection 51(HS2), S83-S91 (2016). 